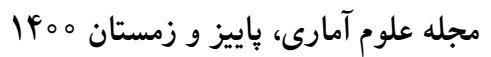

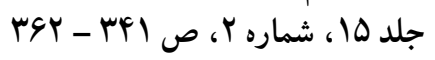

DOI: $10.29252 /$ jss.15.2.341

مقاله بزوهشى

مقايسهاى تصادفى سيستمهاى موازى و سرى متشكل از مولفههاى مقياس با جندين

دورافتاده

$$
\begin{aligned}
& \text { ابراهيم امينىسرشت' و قباد برمالزن † } \\
& \text { ا كروه آمار، دانشعاه بوعلى سينا همدان } \\
& \text { r كروه آمار، دانشكاه زابل }
\end{aligned}
$$

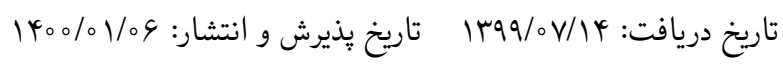

جكيده: در اين مقاله، به مقايسههاى تصادفى سيستمهاى موازى و سرى متشكل از مولفههاى مقياس با

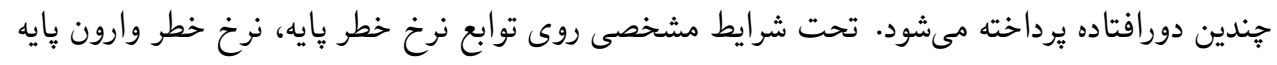

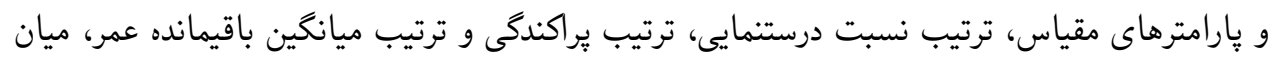

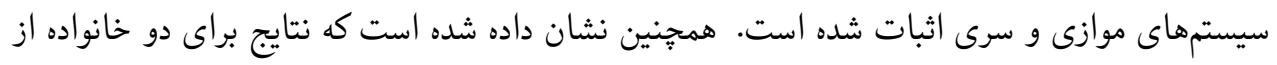

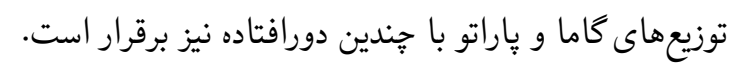

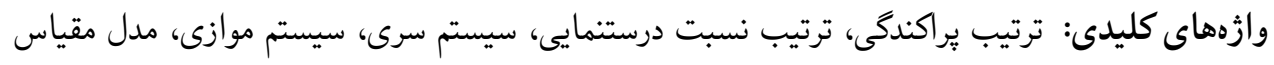
با جندين دورافتاده.

يك خانواده كلى از توزيعها كه شامل توزيعهاى معروفى مانند نمايى، رايلى، وايبل، گاما، ياراتو و غيره

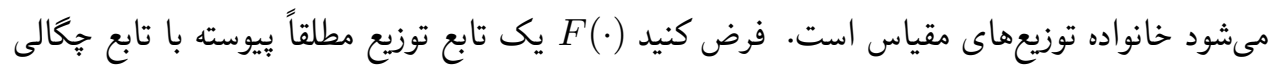

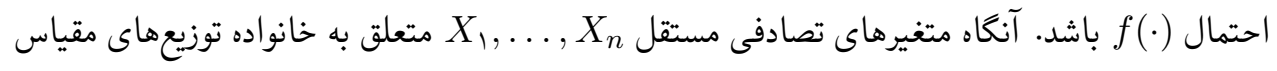


هستند هركاه

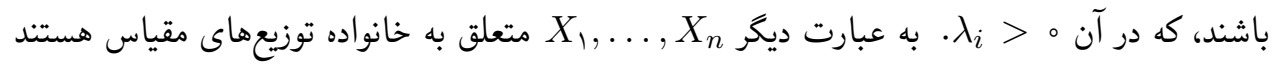
هركاه به ازاى بائد،

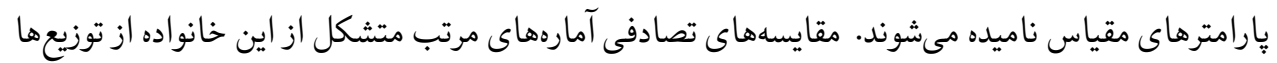

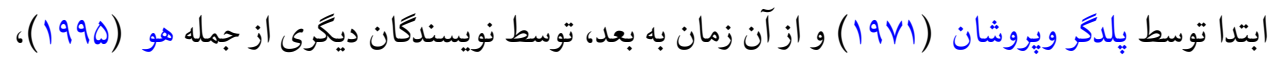

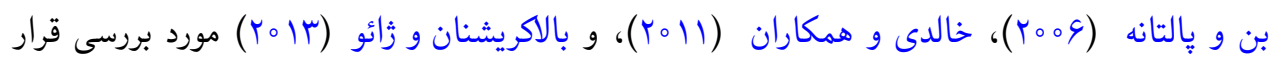

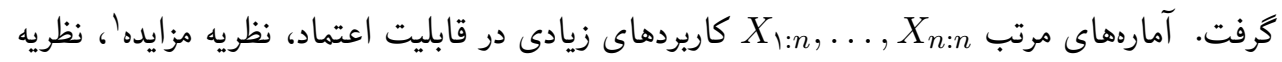

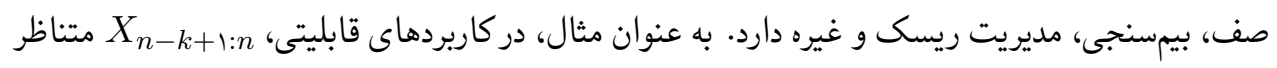

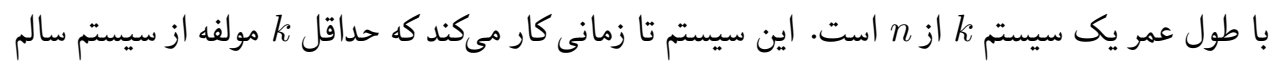
باشد و كار كند. به ويزه،

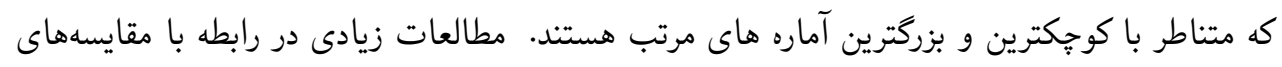

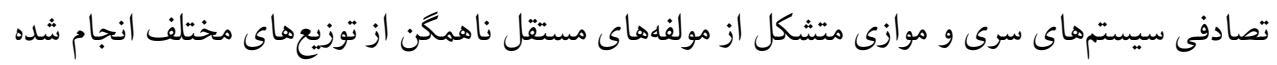

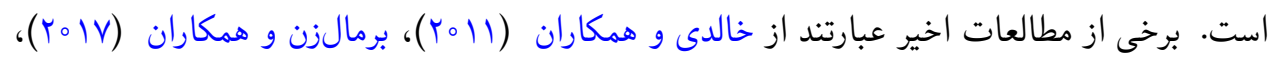

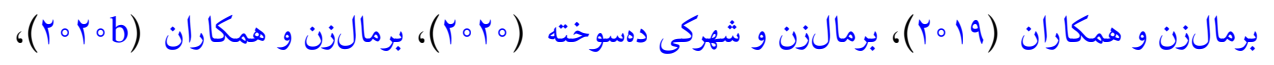

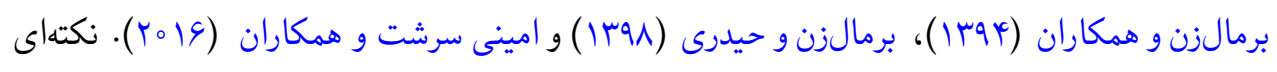

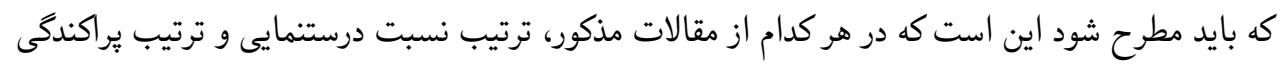

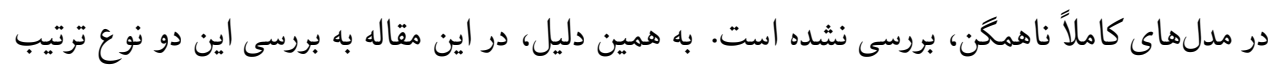
تصادفى برداخته شده است.

اخيراً برخى از نويسندكان به بررسى مقايسه تصادفى سيستمهاى سرى و موازى متشكل از مولفههاى

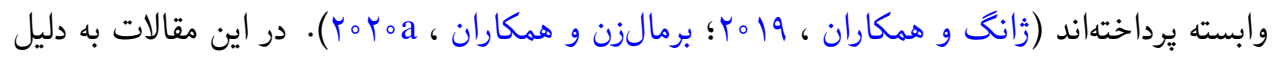

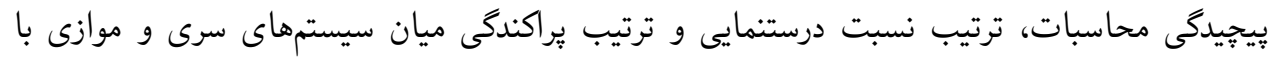
مولفهاى وابسته انجام نشده است. در بسيارى از مواقع، بررسى مقايسه تصادفى سيستمهاى

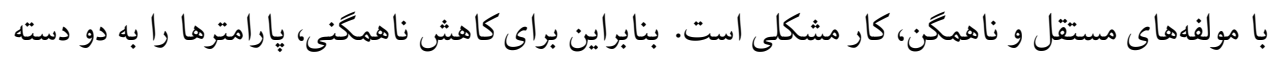

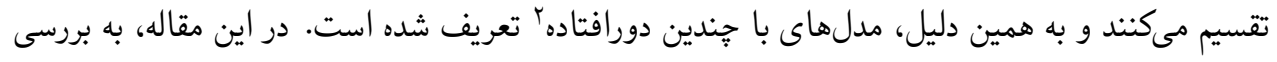

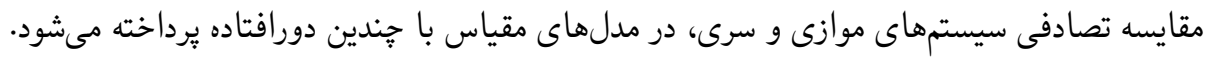
تعريف ا. متغيرهاى تصادفى مستقل ${ }^{1}$ Auction theory

${ }^{2}$ Multiple-outlier models 
rut ابراهيم امينىسرشت و قباد برمالزن .

هركاه داراى تابع توزيع متفاوت ديكرى مانند در رابطه با مقايسه تصادفى سيستمهاى متشكل از مولفهاى با جندين دورافتاده، مىتوان به

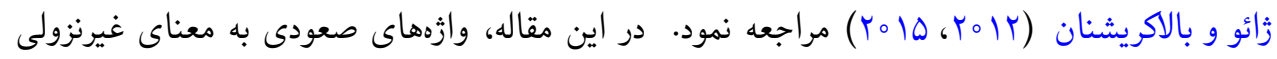

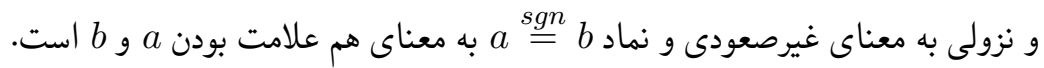

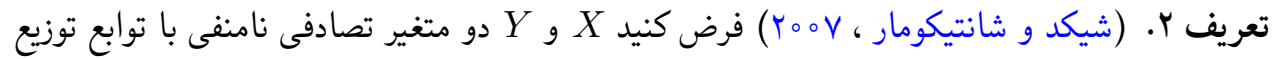

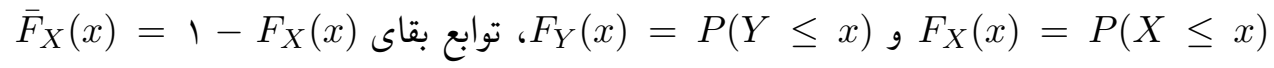
و $r_{X}(x)=1-F_{Y}(x)$ الف الف- X در ترتيب نرخ خطر، بزركتر از $Y$ است (x) صعودى از م $x \geq$

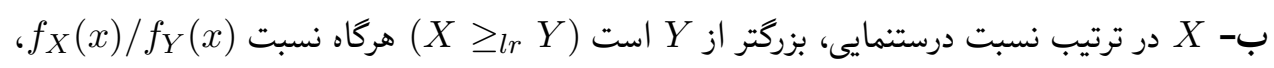

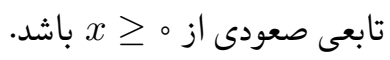

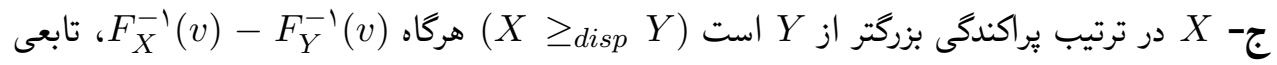

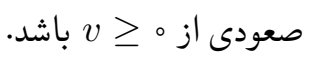

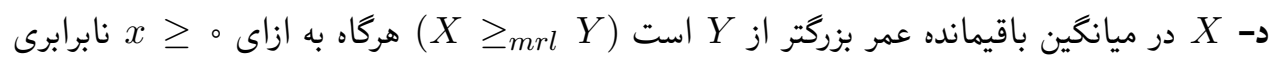

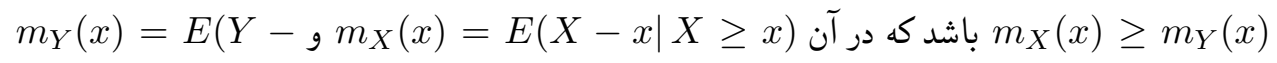
است. $x \mid Y \geq x)$ ميان ترتيبهاى تصادفى مطرح شده، روابط

$$
\begin{aligned}
& X \geq_{l r} Y \Longrightarrow X \geq_{h r} Y \Longrightarrow E(X) \geq E(Y), \\
& X \geq_{\text {disp }} Y \Longrightarrow \operatorname{Var}(X) \geq \operatorname{Var}(Y) .
\end{aligned}
$$

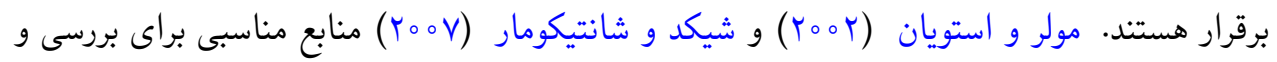

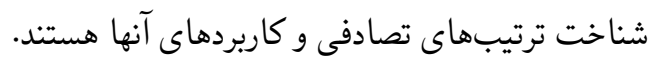
تعريف r. فرض كنيد

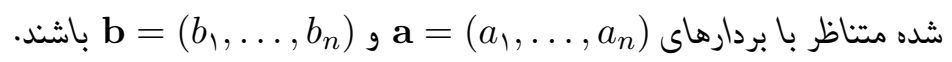




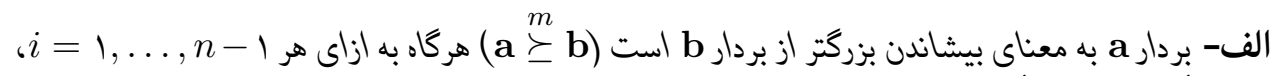
$\cdot \sum_{j=1}^{n} a_{(j)}=\sum_{j=1}^{n} b_{(j)}, \sum_{j=1}^{i} a_{(j)} \leq \sum_{j=1}^{i} b_{(j)}$ ب- بردار a به معناى بيشاندن ضعيف از بالا، بزركتر از بردار b است (a $\cdot \sum_{j=1}^{i} a_{(j)} \leq \sum_{j=1}^{i} b_{(j)} ، i=1, \ldots, n$ ج- بردار a به معناى بيشاندن ضعيف از پايين، بزرگتر از بردار b است (a $\cdot \sum_{j=i}^{n} a_{(j)} \geq \sum_{j=i}^{n} b_{(j)} ، i=1, \ldots, n$ د- بردار a در a $\cdot \prod_{j=1}^{i} a_{(j)} \leq \prod_{j=1}^{i} b_{(j)} ، i=1, \ldots, n$

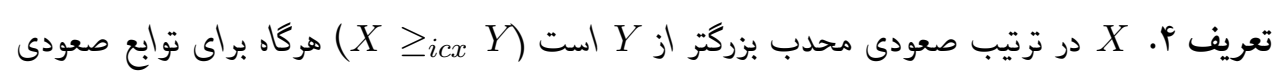

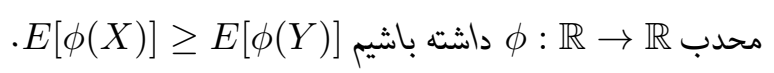

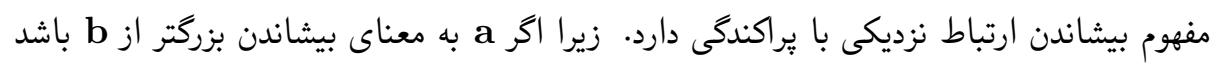

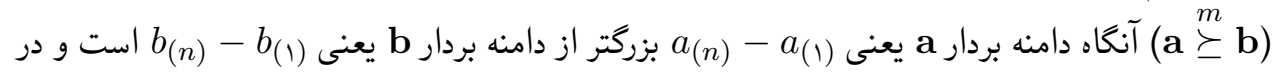

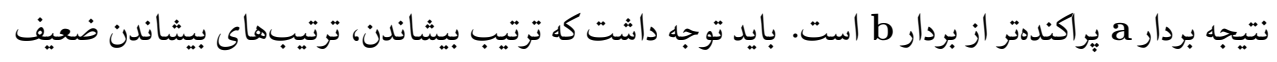

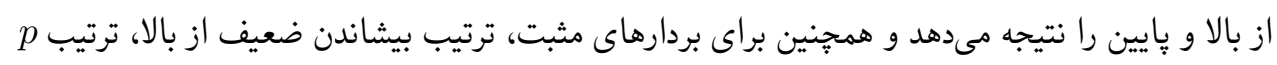

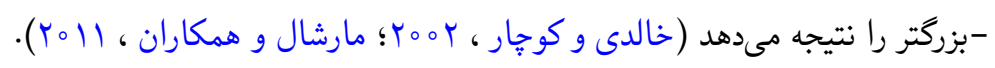
فرض كنيد تابع نرخ خطر يايه (·) باشند. همجنين فرض كنيد

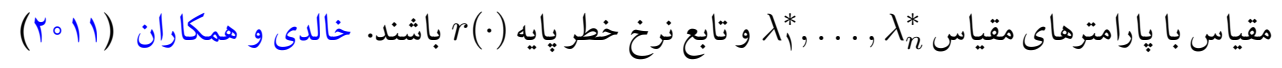
نشان دادند اخر (x)

$$
\left(\lambda_{1}, \ldots, \lambda_{n}\right) \succeq^{m}\left(\lambda_{1}^{*}, \ldots, \lambda_{n}^{*}\right) \Longrightarrow X_{1: n} \geq_{h r} X_{1: n}^{*}
$$

$$
\text { تحت برقرارى رابطه (1) و اينكه (·rr تابعى نزولى باشد، در اين صورت }
$$

$$
\left(\lambda_{1}, \ldots, \lambda_{n}\right) \succeq^{m}\left(\lambda_{1}^{*}, \ldots, \lambda_{n}^{*}\right) \Longrightarrow X_{1: n} \geq_{\text {disp }} X_{1: n}^{*}
$$




$$
\text { بعلاوه اخر (x) xr تابعى نزولى باشد، در اين صورت }
$$$$
\left(\lambda_{1}, \ldots, \lambda_{n}\right) \succeq^{p}\left(\lambda_{1}^{*}, \ldots, \lambda_{n}^{*}\right) \Longrightarrow X_{n: n} \geq_{s t} X_{n: n}^{*}
$$$$
\text { و اخر (x) تابعى نزولى باشد در اين صورت } x^{r} r^{\prime}
$$

$$
\left(\lambda_{1}, \ldots, \lambda_{n}\right) \succeq^{m}\left(\lambda_{1}^{*}, \ldots, \lambda_{n}^{*}\right) \Longrightarrow X_{n: n} \geq_{r h} X_{n: n}^{*}
$$

فرض كنيد يارامترهاى

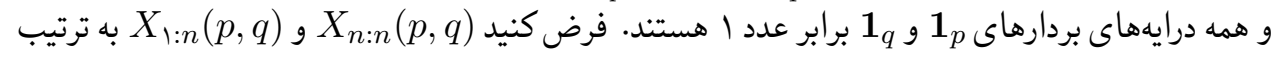
بيانگر طول عمر سيستمهاى موازى و سرى متشكل از اين مولفه هاى مقياس با جندين دورافتاده باشند. تحت فرضهاى فوق و جندين محدوديت روى توابع نرخ خطر وارون يايه و نرخ خطر يايه، در رابطه با سيستمهاى موازى، روابط

$$
\begin{aligned}
& \lambda_{r}^{*}=\lambda_{r}=\lambda \quad, \quad \lambda \geq \lambda_{1}^{*} \geq \lambda_{1} \Longrightarrow X_{n: n}(p, q) \geq_{l r[\operatorname{disp}]} X_{n: n}^{*}(p, q), \\
& \left(\lambda_{1}, \lambda_{r}\right) \succeq^{p}\left(\lambda_{1}^{*}, \lambda_{r}^{*}\right) \Longrightarrow X_{n: n} \geq_{\text {disp }} X_{n: n}^{*}, \\
& X_{1} \geq_{m r l} X_{1}^{*} \quad, \quad \lambda \geq \max \left\{\lambda_{1}, \lambda_{1}^{*}\right\} \Longrightarrow X_{n: n}(p, q) \geq_{m r l} X_{n: n}^{*}(p, q) .
\end{aligned}
$$

اثبات شده است. در رابطه با سيستمهاى سرى، تحت جندين محدوديت روى توابع نرخ خطر يايه، روابط

$$
\begin{aligned}
& \lambda \geq \lambda_{1}^{*} \geq \lambda_{1} \Longrightarrow X_{1: n}(p, q) \geq_{\operatorname{lr}[\operatorname{disp}]} X_{1: n}^{*}(p, q), \\
& \left(\lambda_{1}, \lambda_{r}\right) \succeq^{p}\left(\lambda_{1}^{*}, \lambda_{Y}^{*}\right) \Longrightarrow X_{1: n} \geq_{\text {disp }} X_{1: n}^{*} .
\end{aligned}
$$

نيز حاصل شده است. همجنين از توزيع كاما و پاراتو با جندين دور افتاده، به عنوان مصداقهايى از نتايج اثبات شده، استفاده شده است. مقايسههاى تصادفى سيستمهاى موازى متشكل از مولفههاى مدل مقياس با جندين دورافتاده، از لحاظ ترتيب تصادفى نسبت درستنمايى، ترتيب براكندگى و ترتيب ميانگين باقيمانده عمر در بخش ץ انجام شده است. در بخش ب به بررسى ترتيب تصادفى نسبت درستنمايى و ترتيب 
مقايسهاى تصادفى سيستمهاى موازى و سرى

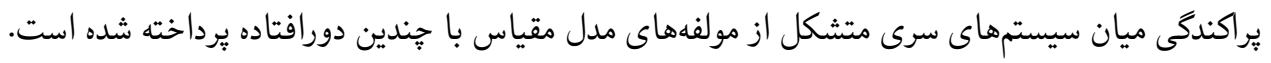

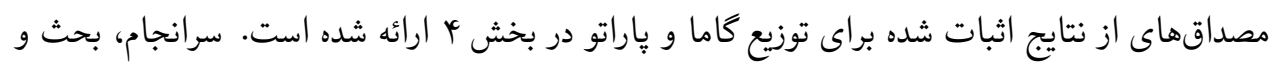

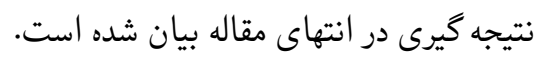

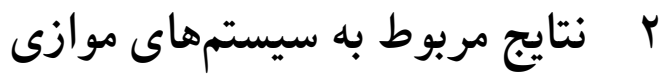

در اين بخش، به مقايسهاى تصادفى سيستهاى موازى متشكل از مولفههاى مقياس با جندين درئ دورافتاده،

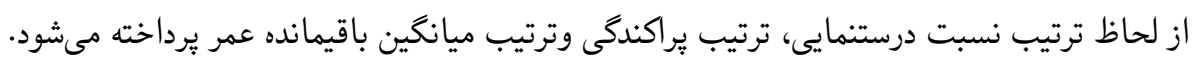

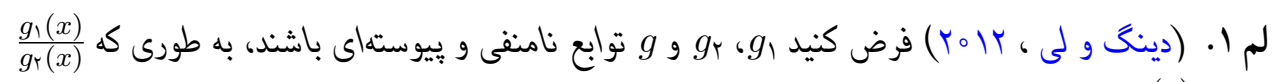

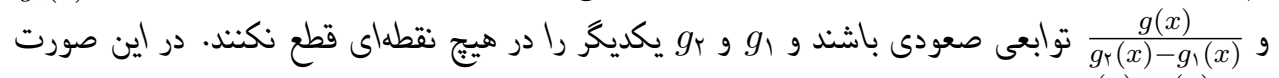
تابع

قضيه l. فرض كنيد دورافتاده با يارامترهاى كن

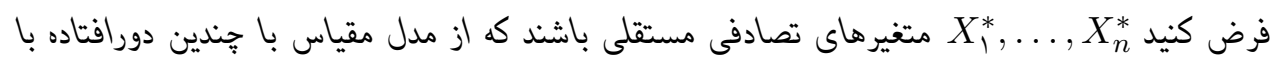

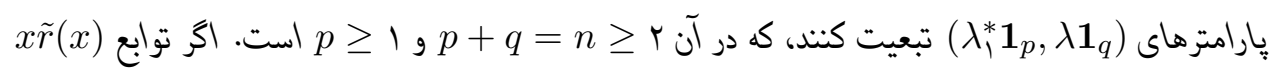
و بارايزماي (x)

$$
\lambda \geq \lambda_{1}^{*} \geq \lambda_{1} \Longrightarrow X_{n: n}(p, q) \geq_{l r} X_{n: n}^{*}(p, q) .
$$

برهان: توابع جكالى احتمال (X)

$$
\begin{array}{ll}
f_{X_{n: n}(p, q)}(t)=[F(\lambda, t)]^{p}[F(\lambda t)]^{q}\left[p \lambda_{1} r\left(\lambda_{1} t\right)+q \lambda r(\lambda t)\right], & t>\circ, \\
f_{X_{n: n}^{*}(p, q)}(t)=\left[F\left(\lambda_{\uparrow}^{*} t\right)\right]^{p}[F(\lambda t)]^{q}\left[p \lambda_{\uparrow}^{*} r\left(\lambda_{\uparrow}^{*} t\right)+q \lambda r(\lambda t)\right], & t>\circ .
\end{array}
$$


ruv ابراهيم امينىسرشت و قباد برمالزن .

$$
\begin{aligned}
& \text { هستند. براى رسيدن به نتيجه لازم، كافى است نشان داده شود به ازاى > t t، } \\
& \frac{f_{X_{n: n}(p, q)}(t)}{f_{X_{n: n}^{*}(p, q)}(t)}=\frac{\left[F\left(\lambda_{1} t\right)\right]^{p}}{\left[F\left(\lambda_{1}^{*} t\right)\right]^{p}} \frac{p \lambda_{1} r\left(\lambda_{1} t\right)+q \lambda r(\lambda t)}{p \lambda_{\uparrow}^{*} r\left(\lambda_{\uparrow}^{*} t\right)+q \lambda r(\lambda t)} \\
& =\Upsilon(t) \frac{p \lambda_{1} r\left(\lambda_{1} t\right)+q \lambda r(\lambda t)}{p \lambda_{1}^{*} r\left(\lambda_{1}^{*} t\right)+q \lambda r(\lambda t)}
\end{aligned}
$$

تابعى صعودى از t است، كه در آن

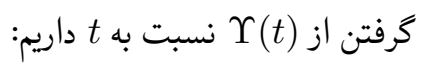

$$
\begin{aligned}
& \Upsilon^{\prime}(t) \stackrel{s g n}{=} \quad p \lambda_{1}\left[F\left(\lambda_{1} t\right)\right]^{p-1} f\left(\lambda_{1} t\right)\left[F\left(\lambda_{1}^{*} t\right)\right]^{p}-p \lambda_{1}^{*}\left[F\left(\lambda_{1}^{*} t\right)\right]^{p-1} f\left(\lambda_{1}^{*} t\right)\left[F\left(\lambda_{1} t\right)\right]^{p} \\
& \stackrel{s g n}{=} \quad \lambda_{1} \tilde{r}(\lambda, t)-\lambda_{1}^{*} \tilde{r}\left(\lambda_{1}^{*} t\right) \text {. }
\end{aligned}
$$

كه در آن 足 $\leq \lambda_{1} \leq \lambda_{1}^{*}$

$$
\Upsilon^{\prime}(t) \stackrel{s g n}{=} \frac{1}{t}\left[x_{\uparrow} \tilde{r}\left(x_{\uparrow}\right)-x_{\curlyvee} \tilde{r}\left(x_{\curlyvee}\right)\right] \geq \circ
$$

كه نامنفى بودن آن از نزولى بودن (x) براساس لم ا، كافى است نشان داده شود

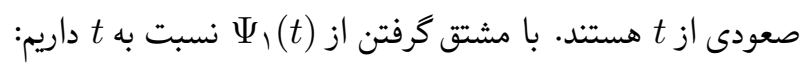

$$
\begin{aligned}
\Psi_{1}^{\prime}(t) & \stackrel{s g n}{=} \quad \lambda_{1} \tilde{r}^{\prime}\left(\lambda_{1} t\right) \tilde{r}\left(\lambda_{1}^{*} t\right)-\lambda_{\uparrow}^{*} \tilde{r}^{\prime}\left(\lambda_{\uparrow}^{*} t\right) \tilde{r}\left(\lambda_{1} t\right) \\
& \stackrel{s g n}{=} \quad \lambda_{1} \frac{\tilde{r}^{\prime}\left(\lambda_{1} t\right)}{\tilde{r}\left(\lambda_{\uparrow} t\right)}-\lambda_{1}^{*} \frac{\tilde{r}^{\prime}\left(\lambda_{\uparrow}^{*} t\right)}{\tilde{r}\left(\lambda_{1}^{*} t\right)} \\
& \stackrel{s g n}{=} \frac{x_{\uparrow} \tilde{r}^{\prime}\left(x_{\uparrow}\right)}{\tilde{r}\left(x_{\uparrow}\right)}-\frac{x_{\uparrow}^{*} \tilde{r}^{\prime}\left(x_{\uparrow}^{*}\right)}{\tilde{r}\left(x_{\uparrow}^{*}\right)} \geq \circ
\end{aligned}
$$

نامنفى بودن عبارت آخر، با قرار دادن \ 


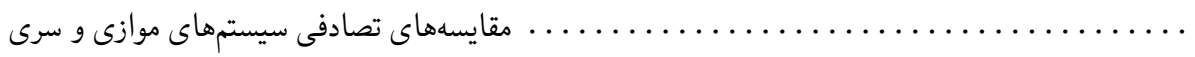

$$
\begin{aligned}
& \text { 政 } \frac{x \tilde{r}^{\prime}(x)}{\tilde{r}(x)} \\
& \Psi_{r}^{\prime}(t) \stackrel{s g n}{=} \quad \lambda_{1}^{*} \lambda\left[\lambda \tilde{r}^{\prime}(\lambda t) \tilde{r}\left(\lambda_{1}^{*} t\right)-\lambda_{1}^{*} \tilde{r}^{\prime}\left(\lambda_{1}^{*} t\right) \tilde{r}(\lambda t)\right] \\
& +\quad \lambda_{1} \lambda\left[\lambda_{1} \tilde{r}^{\prime}(\lambda, t) \tilde{r}(\lambda t)-\lambda \tilde{r}^{\prime}(\lambda t) \tilde{r}(\lambda, t)\right] \\
& \stackrel{s g n}{=} \quad \lambda_{1}^{*} \lambda\left[\lambda \frac{\tilde{r}^{\prime}(\lambda t)}{\tilde{r}(\lambda t)}-\lambda_{1}^{*} \frac{\tilde{r}^{\prime}\left(\lambda_{1}^{*} t\right)}{\tilde{r}\left(\lambda_{1}^{*} t\right)}\right]+\lambda_{1} \lambda\left[\lambda_{1} \frac{\tilde{r}^{\prime}\left(\lambda_{1} t\right)}{\tilde{r}\left(\lambda_{1} t\right)}-\lambda \frac{\tilde{r}^{\prime}(\lambda t)}{\tilde{r}(\lambda t)}\right] \\
& \stackrel{\underline{\underline{g g} n}}{=} \quad x_{\uparrow} x\left[x \frac{\tilde{r}^{\prime}(x)}{\tilde{r}(x)}-x_{\uparrow}^{*} \frac{\tilde{r}^{\prime}\left(x_{\uparrow}^{*}\right)}{\tilde{r}\left(x_{\uparrow}^{*}\right)}\right]+x_{\uparrow} x\left[x_{\uparrow} \frac{\tilde{r}^{\prime}\left(x_{\uparrow}\right)}{\tilde{r}\left(x_{\uparrow}\right)}-x \frac{\tilde{r}^{\prime}(x)}{\tilde{r}(x)}\right] \\
& \geq \quad x_{\uparrow}^{*} x_{\uparrow}\left[x \frac{\tilde{r}^{\prime}(x)}{\tilde{r}(x)}-x_{\uparrow}^{*} \frac{\tilde{r}^{\prime}\left(x_{\uparrow}^{*}\right)}{\tilde{r}\left(x_{\uparrow}^{*}\right)}+x_{\uparrow} \frac{\tilde{r}^{\prime}\left(x_{\uparrow}\right)}{\tilde{r}\left(x_{\uparrow}\right)}-x \frac{\tilde{r}^{\prime}(x)}{\tilde{r}(x)}\right] \\
& =x_{\uparrow}^{*} x_{\uparrow}\left[\frac{x_{\uparrow} \tilde{r}^{\prime}\left(x_{\uparrow}\right)}{\tilde{r}\left(x_{\uparrow}\right)}-\frac{x_{\uparrow}^{*} \tilde{r}^{\prime}\left(x_{\uparrow}^{*}\right)}{\tilde{r}\left(x_{\uparrow}^{*}\right)}\right] \geq \circ,
\end{aligned}
$$

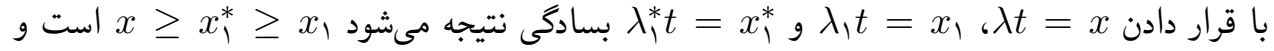

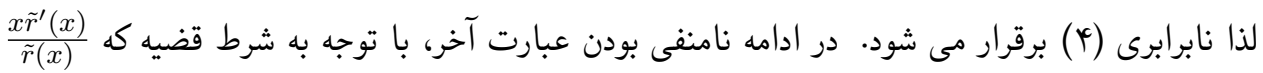

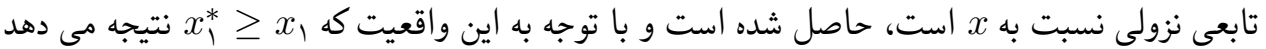

$$
\frac{x_{1} \tilde{r}^{\prime}\left(x_{\curlywedge}\right)}{\tilde{r}\left(x_{1}\right)} \geq \frac{x_{1}^{*} \tilde{r}^{\prime}\left(x_{1}^{*}\right)}{\tilde{r}\left(x_{\uparrow}^{*}\right)}
$$

تذكر l. در قضيه l، اخر شرط الر

$$
\text { قضيه تغيير نكند، آنكاه جهت ترتيب تصادفى نسبت درستنمايى، برعكس مى شئرد }
$$

لم r. (ساندرز و موران ، 19V^) فرض كنيد

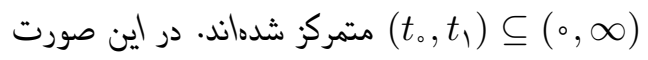

$$
F_{a} \leq_{\text {disp }} F_{a^{*}}, \quad a, a^{*} \in \mathbb{R}, a \leq a^{*},
$$

اخر و فقط اخر

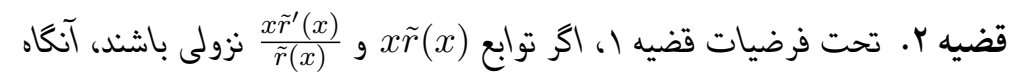

$$
\lambda \geq \lambda_{1}^{*} \geq \lambda_{1} \Longrightarrow X_{n: n}(p, q) \geq_{\text {disp }} X_{n: n}^{*}(p, q) .
$$

برهان: بدون از دست رفتن كليت مساله، فرض كنيد إ 


$$
\begin{aligned}
& F_{n: n, a}(t)=[F((\lambda-a) t)]^{p}[F(\lambda t)]^{q}, \quad t>\circ, \\
& f_{n: n, a}(t)=[F((\lambda-a) t)]^{p}[F(\lambda t)]^{q}[(\lambda-a) \operatorname{pr}((\lambda-a) t)+\lambda q r(\lambda t)], \quad t>\circ,
\end{aligned}
$$$$
\text { هستند. با مشتق گرفتن از }
$$$$
F_{n: n, a}^{\prime}=-t \cdot \tilde{r}((\lambda-a) t)[F((\lambda-a) t)]^{p}[F(\lambda t)]^{q} .
$$$$
\text { كه در آن }
$$

$$
-\frac{F_{n: n, a}^{\prime}(t)}{f_{n: n, a}(t)}=\frac{t}{(\lambda-a) p+\lambda q \nu(t)}
$$

تابعى صعودى از t است، كه در آن

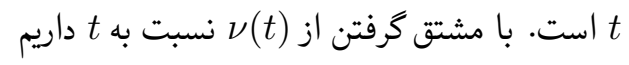

$$
\begin{aligned}
\nu^{\prime}(t) \stackrel{\text { sgn }}{=} & \lambda \tilde{r}^{\prime}(\lambda t) \tilde{r}((\lambda-a) t)-(\lambda-a) \tilde{r}^{\prime}((\lambda-a) t) \tilde{r}(\lambda t) \\
& \stackrel{s g n}{=} \quad \lambda \frac{\tilde{r}^{\prime}(\lambda t)}{\tilde{r}(\lambda t)}-(\lambda-a) \frac{\tilde{r}^{\prime}((\lambda-a) t)}{\tilde{r}((\lambda-a) t)}
\end{aligned}
$$

فرض كنيد

$$
\nu^{\prime}(t) \stackrel{s g n}{=} \frac{x_{\curlyvee} \tilde{r}^{\prime}\left(x_{\Upsilon}\right)}{\tilde{r}\left(x_{\curlyvee}\right)}-\frac{x_{\uparrow} \tilde{r}^{\prime}\left(x_{\uparrow}\right)}{\tilde{r}\left(x_{\uparrow}\right)} \leq \circ
$$

$$
\text { كه نامثبت بودن آن براساس شرط نزولى بودن }
$$

قضيه r. فرض كنيد دورافتاده با بارامترهاى همجنين فرض كنيد با پيارامترهاى 


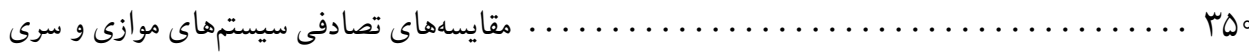

توابع

$$
\lambda_{1} \leq \lambda_{1}^{*} \leq \lambda_{r}^{*} \leq \lambda_{r} \Longrightarrow X_{n: n}(p, q) \geq_{\operatorname{disp}} X_{n: n}^{*}(p, q)
$$

برهان: حالت اول: فرض كنيد فرض كنيد $X_{n: n}(p, q)$ (t) با (t) كافى است نشان داده شود احتمال (X)

$$
\begin{aligned}
& F_{\lambda, n: n}(t)=\left[F\left(\frac{a}{\lambda} t\right)\right]^{p}[F(\lambda t)]^{q}, \quad t>0 . \\
& f_{\lambda, n: n}(t)=\left[F\left(\frac{a}{\lambda} t\right)\right]^{p}[F(\lambda t)]^{q}\left[p \frac{a}{\lambda} \tilde{r}\left(\frac{a}{\lambda} t\right)+q \lambda \tilde{r}(\lambda t)\right], \quad t>\circ . \\
& \text { با مشتق گرفتن از (x) } \\
& F_{\lambda, n: n}^{\prime}(x)=\left[F\left(\frac{a}{\lambda} t\right)\right]^{p}[F(\lambda t)]^{q}\left[-p \frac{a}{\lambda^{r}} t \tilde{r}\left(\frac{a}{\lambda} t\right)+t q \tilde{r}(\lambda t)\right] \\
& \text { بنابراين كافى است نشان داده شود } \\
& -\frac{F_{\lambda, n: n}^{\prime}(t)}{f_{\lambda, n: n}(t)}=\frac{p \frac{a}{\lambda^{r}} t \tilde{r}\left(\frac{a}{\lambda} t\right)-t q \tilde{r}(\lambda t)}{p \frac{a}{\lambda} \tilde{r}\left(\frac{a}{\lambda} t\right)+q \lambda \tilde{r}(\lambda t)} \stackrel{s g n}{=} t \frac{p \frac{a}{\lambda} t \tilde{r}\left(\frac{a}{\lambda} t\right)-q \lambda t \tilde{r}(\lambda t)}{p \frac{a}{\lambda} t \tilde{r}\left(\frac{a}{\lambda} t\right)+q \lambda t \tilde{r}(\lambda t)}
\end{aligned}
$$

تابعى صعودى از t است. با توجه به نزولى بودن (†)

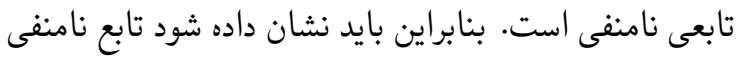

$$
\psi(t)=\frac{p \frac{a}{\lambda} t \tilde{r}\left(\frac{a}{\lambda} t\right)-q \lambda t \tilde{r}(\lambda t)}{p \frac{a}{\lambda} t \tilde{r}\left(\frac{a}{\lambda} t\right)+q \lambda t \tilde{r}(\lambda t)}=\frac{p \frac{a}{\lambda}-q \lambda v(t)}{p \frac{a}{\lambda}+q \lambda v(t)}
$$

تابعى صعودى از t است، كه در آن 
rol ابراهيم امينىسرشت و قباد برمالزن . است، اخر و فقط اخر (t) تابعى نزولى از t باشد. با مشتق گرفتن از (t) نسبت به t داريم:

$$
v^{\prime}(t) \stackrel{s g n}{=} \frac{\lambda \tilde{r}^{\prime}(\lambda t)}{\tilde{r}(\lambda t)}-\frac{\frac{a}{\lambda} \tilde{r}^{\prime}\left(\frac{a}{\lambda} t\right)}{\tilde{r}\left(\frac{a}{\lambda} t\right)}
$$

با قرار دادن \

$$
v^{\prime}(t) \stackrel{\operatorname{sgn}}{=} \frac{x_{\curlyvee} \tilde{r}^{\prime}\left(x_{\Upsilon}\right)}{\tilde{r}\left(x_{\curlyvee}\right)}-\frac{x_{\uparrow} \tilde{r}^{\prime}\left(x_{\uparrow}\right)}{\tilde{r}\left(x_{\uparrow}\right)} \leq \circ
$$

كه نامثبت بودن عبارت آخر، از شرط نزولى بودن حالت دوم: فرض كنيد و و حالت دوم: $\theta \lambda_{r}=\lambda_{r}^{*} \lambda_{r}^{*}$ مان

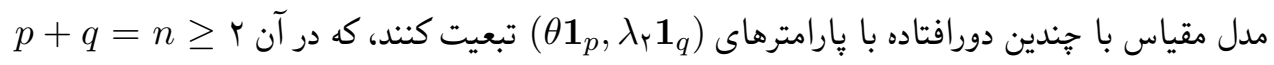

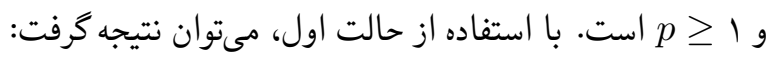

$$
Z_{n: n}(p, q) \geq_{\text {disp }} X_{n: n}^{*}(p, q)
$$

جون I

$$
X_{n: n}(p, q) \geq_{\text {disp }} Z_{n: n}(p, q)
$$

با استفاده از نابرابرىهاى (V) و (^) نتيجه لازم حاصل مىشود. فرع ا. فرض كنيد با بارامترهاى

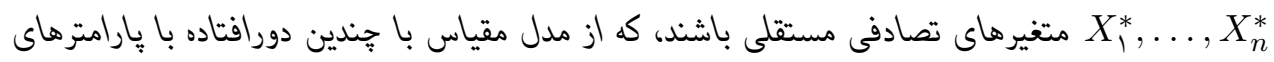
و $\left(\lambda \mathbf{1}_{p}, \lambda \mathbf{1}_{q}\right)$

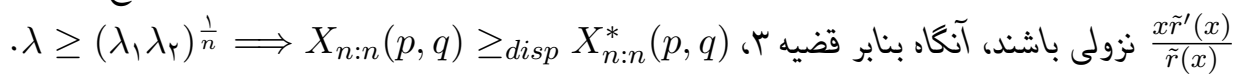
لم r. (بارلو و يروشان ، 1999) فرض كنيد (x) يك اندازه لبخ-اشتليس، نه لزوماً مثبت، باشد به 
طورى كه به ازاى > $t$ نابرابرى ه

$$
\text { در اين صورت }
$$

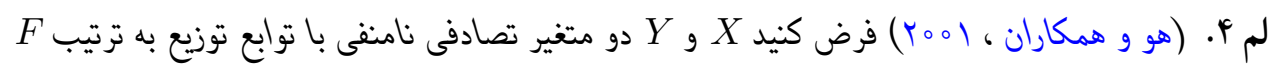

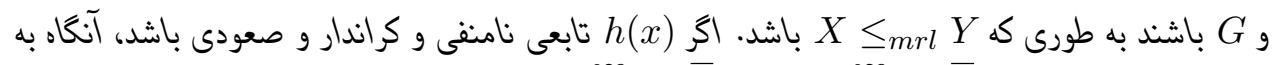

$$
\frac{\int_{t}^{\infty} h(x) \bar{F}(x) d x}{\bar{F}(t)} \leq \frac{\int_{t}^{\infty} h(x) \bar{G}(x) d x}{\bar{G}(t)}
$$

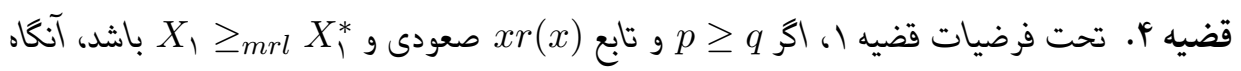

$$
\lambda \geq \max \left\{\lambda_{1}, \lambda_{1}^{*}\right\} \Longrightarrow X_{n: n}(p, q) \geq_{m r l} X_{n: n}^{*}(p, q)
$$

برهان: حالت اول: فرض كنيد و

$$
\begin{aligned}
m_{X_{n: n}(p, q)}(t) & =\frac{\int_{t}^{\infty}\left(1-[F(\lambda, x)]^{p}[F(\lambda x)]^{q}\right) d x}{1-[F(\lambda, t)]^{p}[F(\lambda t)]^{q}} \\
& =\frac{\int_{t}^{\infty}\left(\bar{F}_{X_{q: q}}(x)+\bar{F}_{X_{p: p}}(x) F_{X_{q: q}}(x)\right) d x}{\bar{F}_{X_{q: q}}(t)+\bar{F}_{X_{p: p}}(t) F_{X_{q: q}}(t)}, \\
m_{X_{n: n}^{*}(p, q)}(t) & =\frac{\int_{t}^{\infty}\left(\bar{F}_{X_{q: q}}(x)+\bar{F}_{X_{p: p}^{*}}(x) F_{X_{q: q}}(x)\right) d x}{\bar{F}_{X_{q: q}}(t)+\bar{F}_{X_{p: p}^{*}}(t) F_{X_{q: q}}(t)} .
\end{aligned}
$$

كافى است نشان داده شود ه

$$
\begin{aligned}
\Psi(t) & \stackrel{\text { sgn }}{=}\left[\bar{F}_{X_{q: q}}(t)+\bar{F}_{X_{p: p}^{*}}(t) F_{X_{q: q}}(t)\right]\left[\int_{t}^{\infty}\left(\bar{F}_{X_{q: q}}(x)+\bar{F}_{X_{p: p}}(x) F_{X_{q: q}}(x)\right) d x\right] \\
& -\left[\bar{F}_{X_{q: q}}(t)+\bar{F}_{X_{p: p}}(t) F_{X_{q: q}}(t)\right]\left[\int_{t}^{\infty}\left(\bar{F}_{X_{q: q}}(x)+\bar{F}_{X_{p: p}^{*}}(x) F_{X_{q: q}}(x)\right) d x\right] \\
& =\bar{F}_{X_{q: q}}(t) \int_{0}^{\infty}\left[\bar{F}_{X_{p: p}}(x)-\bar{F}_{X_{p: p}^{*}}(x)\right] F_{X_{q: q}}(x) d x \\
& +\bar{F}_{X_{p: p}^{*}}(t) F_{X_{q: q}}(t) \int_{t}^{\infty}\left(\bar{F}_{X_{q: q}}(x)+\bar{F}_{X_{p: p}}(x) F_{X_{q: q}}(x)\right) d x \\
& -\bar{F}_{X_{p: p}}(t) F_{X_{q: q}}(t) \int_{t}^{\infty}\left(\bar{F}_{X_{q: q}}(x)+\bar{F}_{X_{p: p}^{*}}(x) F_{X_{q: q}}(x)\right) d x
\end{aligned}
$$


ror ابراهيم امينىسرشت و قباد برمالزن .

$$
\begin{aligned}
& \geq \bar{F}_{X_{q: q}}(t) \int_{\circ}^{\infty}\left[\bar{F}_{X_{p: p}}(x)-\bar{F}_{X_{p: p}^{*}}(x)\right] F_{X_{q: q}}(x) d x \\
& +\bar{F}_{X_{p: p}}(t) F_{X_{q: q}}(t) \int_{t}^{\infty}\left(\bar{F}_{X_{q: q}}(x)+\bar{F}_{X_{p: p}}(x) F_{X_{q: q}}(x)\right) d x \\
& -\bar{F}_{X_{p: p}}(t) F_{X_{q: q}}(t) \int_{t}^{\infty}\left(\bar{F}_{X_{q: q}}(x)+\bar{F}_{X_{p: p}^{*}}(x) F_{X_{q: q}}(x)\right) d x \\
& =\bar{F}_{X_{q: q}}(t) \int_{t}^{\infty}\left[\bar{F}_{X_{p: p}}(x)-\bar{F}_{X_{p: p}^{*}}(x)\right] F_{X_{q: q}}(x) d x \\
& +\bar{F}_{X_{p: p}}(t) F_{X_{q: q}}(t) \int_{t}^{\infty}\left[\bar{F}_{X_{p: p}}(x)-\bar{F}_{X_{p: p}^{*}}(x)\right] F_{X_{q: q}}(x) d x \\
& \geq \circ,
\end{aligned}
$$

كه در آن نابرابرى (q) با استفاده از (q)

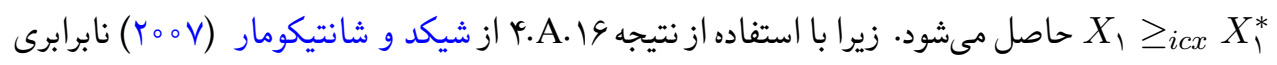
تصادفى

$$
\int_{t}^{\infty}\left[\bar{F}_{X_{p: p}}(x)-\bar{F}_{X_{p: p}^{*}}(x)\right] d x \geq \circ .
$$$$
\text { همواره برقرار است. نابرابرى (10) نيز با استفاده از لم ب حاصل مىشود. }
$$
حالت دوم: فرض كنيد (t)

$$
\begin{gathered}
m_{X_{n: n}(p, q)}(t)=\frac{\int_{t}^{\infty}\left[\bar{F}(\lambda x) \sum_{j=0}^{q-1} F^{j}(\lambda x)+\left(1-F^{p}(\lambda, x)\right) F^{q}(\lambda x)\right] d x}{\bar{F}(\lambda t) \sum_{j=0}^{q-1} F^{j}(\lambda t)+\left(1-F^{p}(\lambda, t)\right) F^{q}(\lambda t)} . \\
\bar{F}_{X_{p: p}}(t) \Psi(t) \stackrel{s g n}{=} \bar{F}_{X_{p: p}}(t) F^{q}(\lambda t)\left[\bar{F}_{X_{p: p}^{*}}(t)-\bar{F}_{X_{p: p}}(t)\right] \int_{t}^{\infty} \bar{F}(\lambda x) \sum_{j=0}^{q-1} F^{j}(\lambda x) d x \\
+\bar{F}_{X_{p: p}}(t)\left[\bar{F}(\lambda t) \sum_{j=0}^{q-1} F^{j}(\lambda t)+\bar{F}_{X_{p: p}^{*}}(t) F^{q}(\lambda t)\right] \int_{t}^{\infty} \bar{F}_{X_{p: p}}(x) F^{q}(\lambda x) d x
\end{gathered}
$$




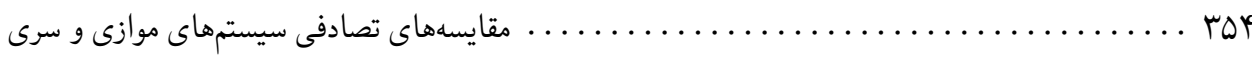

$$
\begin{aligned}
& -\bar{F}_{X_{p: p}}(t)\left[\bar{F}(\lambda t) \sum_{j=\circ}^{q-1} F^{j}(\lambda t)+\bar{F}_{X_{p: p}}(t) F^{q}(\lambda t)\right] \int_{t}^{\infty} \bar{F}_{X_{p: p}^{*}}(x) F^{q}(\lambda x) d x \\
& \geq \bar{F}_{X_{p: p}}(t) F^{q}(\lambda t)\left[\bar{F}_{X_{p: p}^{*}}(t)-\bar{F}_{X_{p: p}}(t)\right] \int_{t}^{\infty} \bar{F}(\lambda x) \sum_{j=0}^{q-1} F^{j}(\lambda x) d x \\
& +\bar{F}_{X_{p: p}}(t) \bar{F}(\lambda t) \sum_{j=0}^{q-1} F^{j}(\lambda t) \int_{t}^{\infty} \bar{F}_{X_{p: p}}(x) F^{q}(\lambda x) d x \\
& -\bar{F}_{X_{p: p}}(t) \bar{F}(\lambda t) \sum_{j=0}^{q-1} F^{j}(t) \int_{t}^{\infty} \bar{F}_{X_{p: p}^{*}}(x) F^{q}(\lambda x) d x \\
& \geq \bar{F}_{X_{p: p}}(t) F^{q}(\lambda t)\left[\bar{F}_{X_{p: p}^{*}}(t)-\bar{F}_{X_{p: p}}(t)\right] \int_{t}^{\infty} \bar{F}(\lambda x) \sum_{j=。}^{q-1} F^{j}(\lambda x) d x \\
& +\quad \bar{F}_{X_{p: p}}(t) \bar{F}(\lambda t) \sum_{j=0}^{q-1} F^{j}(t) \int_{t}^{\infty} \bar{F}_{X_{p: p}}(x) F^{q}(\lambda x) d x \\
& -\bar{F}_{X_{p: p}^{*}}(t) \bar{F}(\lambda t) \sum_{j=0}^{q-1} F^{j}(t) \int_{t}^{\infty} \bar{F}_{X_{p: p}}(x) F^{q}(\lambda x) d x \\
& =\bar{F}_{X_{p: p}}(t) F^{q}(\lambda t)\left[\bar{F}_{X_{p: p}^{*}}(t)-\bar{F}_{X_{p: p}}(t)\right] \int_{t}^{\infty} \bar{F}(\lambda x) \sum_{j=0}^{q-1} F^{j}(\lambda x) d x \\
& +\bar{F}(\lambda t) \sum_{j=\circ}^{q-1} F^{j}(\lambda t)\left[\bar{F}_{X_{p: p}}(t)-\bar{F}_{X_{p: p}^{*}}(t)\right] \int_{t}^{\infty} \bar{F}_{X_{p: p}}(x) F^{q}(\lambda x) d x \\
& \stackrel{s g n}{=} \bar{F}_{X_{q: q}}(t) \int_{t}^{\infty} \bar{F}_{X_{p: p}}(x) F^{q}(\lambda x) d x \\
& -\bar{F}_{X_{p: p}}(t) F^{q}(\lambda t) \int_{t}^{\infty} \bar{F}_{X_{q: q}}(x) d x \\
& \geq F^{q}(\lambda t)\left[\bar{F}_{X_{q: q}}(t) \int_{t}^{\infty} \bar{F}_{X_{p: p}}(x) d x-\bar{F}_{X_{p: p}}(t) \int_{t}^{\infty} \bar{F}_{X_{q: q}}(x) d x\right] \\
& \geq \quad \circ
\end{aligned}
$$

كه در آن اولين و دومين نابرابرى با استفاده از لم ץ حاصل مىشوند. بايد توجه داشت كه با استفاده از شرط صعودى بودن (x) مr مىتوان نتيجه كرفت 
شيكد و شانتيكومار (Y०V) به ترتيت نابرابرىهاى

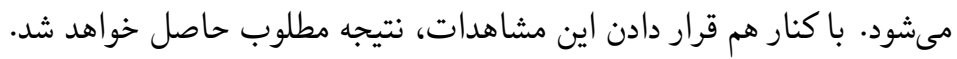

\section{r متايج مربوط به سيستمهاى سرى}

قضيه ه. تحت فرضيات قضيه ا، اخر توابع xr(x) و

$$
\lambda \geq \lambda_{1}^{*} \geq \lambda_{1} \Longrightarrow X_{1: n}(p, q) \geq_{l r} X_{1: n}^{*}(p, q) .
$$

برهان: توابع جگالى احتمال (p,

$$
\begin{aligned}
& f_{X_{1: n}}(p, q)(t)=p \lambda_{1} f\left(\lambda_{1} t\right)\left[\bar{F}\left(\lambda_{1} t\right)\right]^{p-1}[\bar{F}(\lambda t)]^{q}+q \lambda f(\lambda t)\left[\bar{F}\left(\lambda_{1} t\right)\right]^{p}[\bar{F}(\lambda t)]^{q-1} \\
& f_{X_{1: n}^{*}(p, q)}(t)=p \lambda_{1}^{*} f\left(\lambda_{1}^{*} t\right)\left[\bar{F}\left(\lambda_{1}^{*} t\right)\right]^{p-1}[\bar{F}(\lambda t)]^{q}+q \lambda f(\lambda t)\left[\bar{F}\left(\lambda_{1}^{*} t\right)\right]^{p}[\bar{F}(\lambda t)]^{q-1}
\end{aligned}
$$

$$
\text { بنابراين به ازاى ه > t كافى است نشان داده شود }
$$

$$
\frac{f_{X_{1: n}(p, q)}(t)}{f_{X_{1: n}^{*}(p, q)}(t)}=\frac{\left[\bar{F}\left(\lambda_{1} t\right)\right]^{p}}{\left[\bar{F}\left(\lambda_{\uparrow}^{*} t\right)\right]^{p}} \frac{p \lambda_{1} r\left(\lambda_{1} t\right)+q \lambda r(\lambda t)}{p \lambda_{1}^{*} r\left(\lambda_{1}^{*} t\right)+q \lambda r(\lambda t)}
$$

تابعى صعودى از t است، كه در آن (·) تابع نرخ خطر است. با استفاده از شرط إن

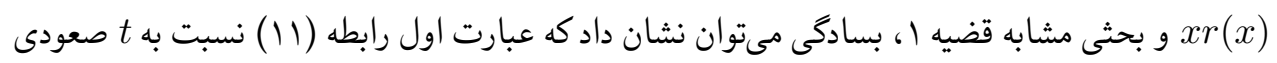

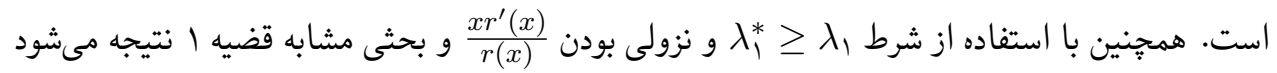
عبارت دوم رابطه (11) نسبت به t صعودى است. بنابراين نتيجه لازم، حاصل مى الشود.

\section{جن إند مصداق براى نتايج اثبات شده}

نكتهاى كه لازم است بيان شود اين است كه شرايط بيان شده در قضاياى بخش ب و ب كاملاً كلى هستند

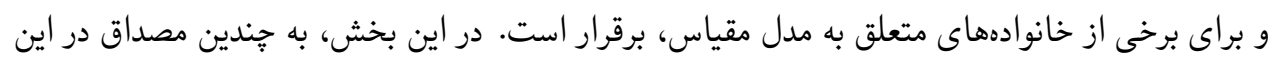
زمينه يرداخته مىشود. 
مقايسهاى تصادفى سيستمهاى موازى و سرى . - مق

لم ه. (ميسرا و وان در ميلون ، بهمب) فرض كنيد $\Theta$ يك زيرمجموعه از اعداد حقيقى و U يك متغير تصادفى نامنفى متعلق به خانواده

$$
\theta_{1} \leq \theta_{\Upsilon} \Longrightarrow H\left(\cdot \mid \theta_{\uparrow}\right) \leq_{s t}\left(\geq_{s t}\right) H\left(\cdot \mid \theta_{\Upsilon}\right)
$$

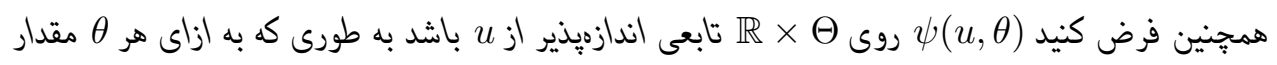
$E_{\theta}[\psi(U, \theta)]$

الف- [ن [ن (نزولى) از $u$ باشد.

ب- [ ]

فرع r. فرض كنيد با بارامترهاى مقياس مقاس

مقياس الف- اكر ب- اخر

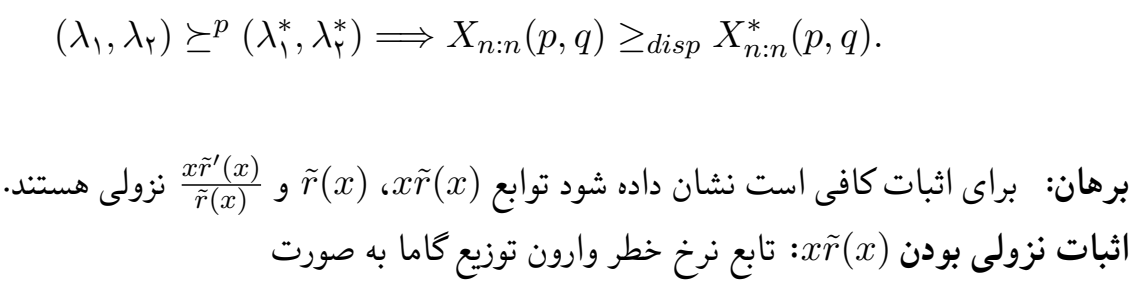
$\tilde{r}(x)=\frac{1}{x \int_{0}^{1} u^{r-1} e^{(1-u) x} d u}$ 


$$
(x \tilde{r}(x))^{\prime}=\frac{-\int_{0}^{1} u^{r-1}(1-u) e^{(1-u) x} d u}{\left(\int_{0}^{1} u^{r-1} e^{(1-u) x} d u\right)^{r}}
$$

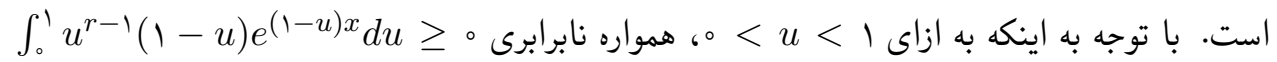

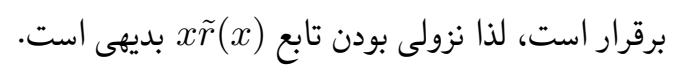
اثبات نزولى بودن (x) همواره تابعى نزولى است. اثبات نزولى بودن

$$
\tilde{r}^{\prime}(x)=-\frac{\int_{0}^{1} u^{r-1} e^{(1-u) x} d u+x \int_{0}^{1} u^{r-1}(1-u) e^{(1-u) x} d u}{\left[x \int_{0}^{1} u^{r-1} e^{(1-u) x} d u\right]^{\Upsilon}} .
$$

$$
\eta(x)=\frac{x r^{\prime}(x)}{r(x)}=-1-x \frac{\int_{0}^{1} u^{r-1}(1-u) e^{(1-u) x} d u}{\int_{0}^{1} u^{r-1} e^{(1-u) x} d u}
$$

جون

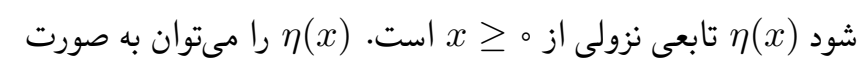

$$
\begin{aligned}
\eta(x) & =-x \int_{0}^{1}(1-u) \frac{u^{r-1} e^{(1-u) x} d u}{\int_{0}^{1} u^{r-1} e^{(1-u) x} d u}-1 \\
& =-x \int_{0}^{1} \psi(u, x) h(u \mid x) d u-1 \\
& =-x E[\psi(U, x)]-1
\end{aligned}
$$

بازنويسى كرد، كه در آن نشان داده شود [E[U 
مقايسهاى تصادفى سيستمهاى موازى و سرى

Uتعلق به خانواده $U$

$$
h(u \mid x)=c(x) u^{r-1} e^{(1-u) x} \quad \circ<u<1,
$$

است، كه در آن (x) ثابت نرمالساز است. واضح است كه (u,x)

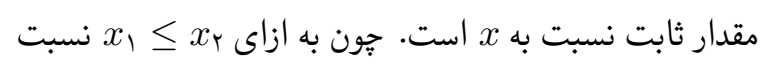

$$
\frac{h\left(u \mid x_{\curlyvee}\right)}{h\left(u \mid x_{\curlywedge}\right)}=\frac{e^{(1-u) x_{\curlyvee}}}{e^{(1-u) x_{\curlywedge}}}=e^{(1-u)\left(x_{\curlyvee}-x_{\curlywedge}\right)}
$$

تابعى نزولى نسبت به $u$ است در نتيجه ( بنابر لم ه، [E

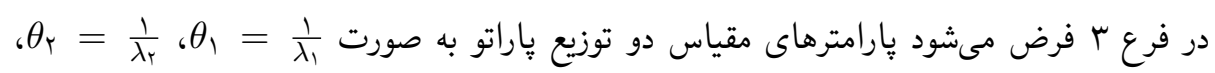

$$
\text { باشبr } \theta_{r}^{*}=\frac{1}{\lambda_{r}^{*}}, \theta_{1}^{*}=\frac{1}{\lambda_{1}^{*}}
$$

فرع r. فرض كنيد

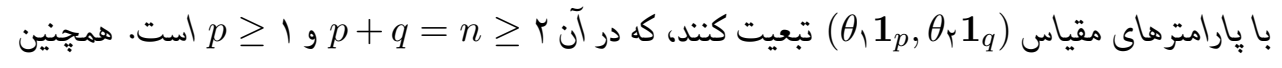
فرض كنيد

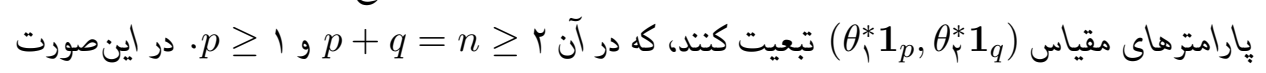
الف- اكر ب- اخ برهان: تابع بقاى توزيع ياراتو عبه صورت

$$
\bar{F}(x)=\left(\frac{\lambda}{x}\right)^{a}, \quad x \geq \lambda \geq \circ, a \geq \circ .
$$

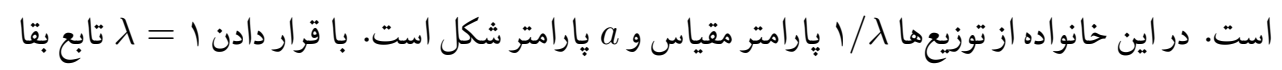

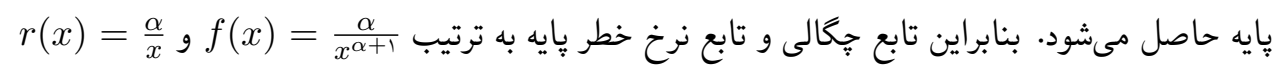

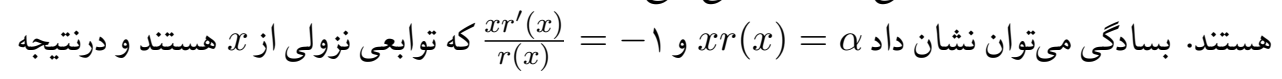
در شرايط قضيه ه صدق مي هن:د. 


\section{بحث و نتيجهكيرى}

در اين مقاله، به مقايسه تصادفى سيستمهاى موازى و سرى متشكل از مولفههاى مقياس با جندين دورافتاده

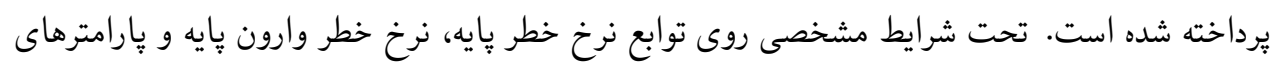

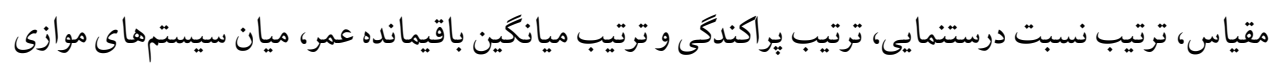

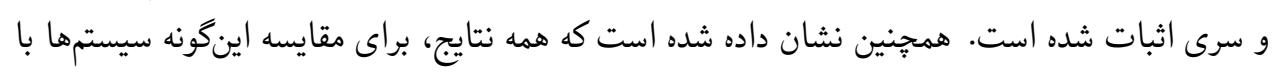

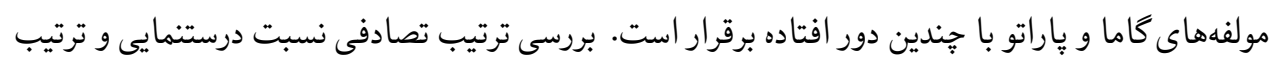

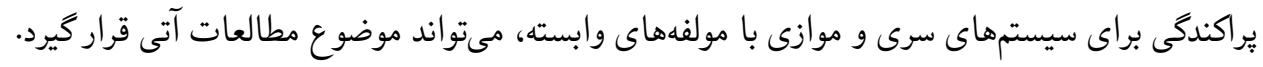

\section{تقدير و تشكر}

نويسندكان از ييشنهادات ارزنده داوران محترم و ويراستار مجله كه باعث ارائه بهتر و افزايش سطح كيفى

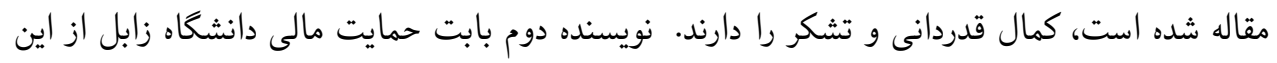

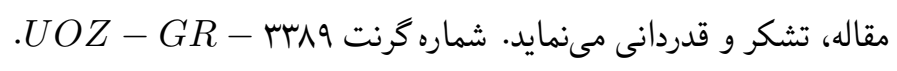

\section{مراجع}

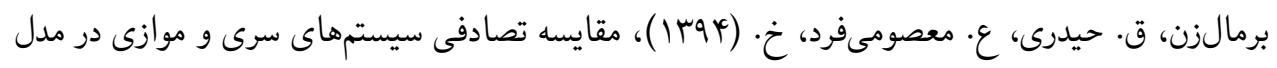

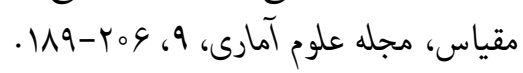

برمالزن، ق. حيدرى. (1 (1) )، مقايسه تصادفى سيستمهاى سرى و موازى با مولفههاى مستقل و ناهمخن

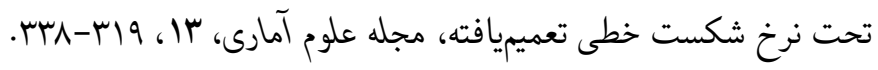

امينى سرشت، ا. برمالزن، ق. (99 (1)، ترتيب نسبت درستنمايى ميان سيستمهاى k از n متشكل از

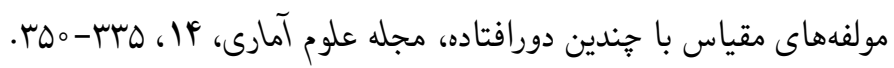

Balakrishnan, N. and Zhao, P. (2013), Ordering Properties of Order Statistics from Heterogeneous Populations: A Review with an Emphasis on Some Recent Developments, Probability in the Engineering and Informational Sciences, 27, 403-443. 
Barmalzan, G., Ayat, S. M. and Balakrishnan, N. (2020a), Stochastic Comparisons of Series and Parallel Systems with Dependent Burr Type XII Components, Communications in Statistics-Theory and Method, DOI: 10.1080/03610926.2020.1772307.

Barmalzan, G., Kosari, S. and Balakrishnan, N. (2020b), Usual Stochastic and Reversed Hazard Orders of Parallel Systems with Independent Heterogeneous Components, Communications in Statistics-Theory and Methods, DOI: 10.1080/03610926.2020.1823415.

Barmalzan, G. and Dehsukhteh, S. S. (2020), Comparisons of Series and Parallel Systems with Heterogeneous Exponentiated Geometric Components, Communications in Statistics-Theory and Methods, DOI: 10.1080/03610926.2020.1716251.

Barmalzan, G., Najafabadi, A. T. P. and Balakrishnan, N. (2017), Orderings for Series and Parallel Systems Comprising Heterogeneous Exponentiated Weibull-Geometric Components, Communications in StatisticsTheory and Methods, 46, 9869-9880.

Barmalzan, G., Najafabadi, A. T. P. and Balakrishnan, N. (2019), Ordering Results for Series and Parallel Systems Comprising Heterogeneous Exponentiated Weibull Components, Communications in Statistics-Theory and Methods, 48, 660-675.

Barlow, R. E. and Proschan, F. (1975), Statistical Theory of Reliability and Life Testing: Probability Models, Silver Spring, Maryland.

Boland, P. J., El-Neweihi, E. and Proschan, F. (1994), Schur properties of convolutions of exponential and geometric random variables. Journal of Multivariate Analysis, ,48, 157-167. 
Bon, J. L. and Paltanea, E. (2006), Comparisons of Order Statistics in a Random Sequence to the Same Statistics with i.i.d. Variables, ESAIM: Probability and Statistics, 10, 1-10.

Ding, W. and Li, X. (2012), The Optimal Allocation of Active Redundancies to $k$-out-of- $n$ Systems with respect to Hazard Rate Ordering, Journal of Statistical Planning and Inference, 142, 1878-1887.

Hu, T. (1995), Monotone Coupling and Stochastic Ordering of Order Statistics, System Science and Mathematical Sciences, 8, 209-214.

Hu, T., Zhu, Z. and Wei, Y. (2001), Likelihood Ratio and Mean Residual Life Order of Order Statistics with Heterogeneous Random Variables, Probability in the Engineering and Informational Sciences, 15, 259-272.

Khaledi, B., Farsinezhad, S. and Kochar, S. C. (2011), Stochastic Comparisons of Order Statistics in the Scale Model, Journal of Statistical Planning and Inference, 141, 276-286.

Khaledi, B. and Kochar, S. C. (2002), Dispersive Ordering among Linear Combinations of Uniform Random Variables, Journal of Statistical Planning and Inference, 100, 13-21.

Marshall, A. W., Olkin, I. and Arnold, B. C. (2011), Inequalities: Theory of Majorization and Its Applications, Springer, New York.

Misra, N. and van der Meulen, E. C. (2003), On Stochastic Properties of M-spacings. Journal of Statistical Planning and Inferences. 115, 683-697.

Müller, A. and Stoyan, D. (2002), Comparison Methods for Stochastic Models and Risks, John Wiley \& Sons, New York. 
Pledger, P. and Proschan, F. (1971), Comparisons of Order Statistics and of Spacings from Heterogeneous Distributions, In: Optimizing Methods in Statistics (Ed., J.S. Rustagi), pp. 89-113, Academic Press, New York.

Saunders, I.W. and Moran, P.A. (1978), On the Quantiles of the Gamma and F Distributions, Journal of Applied Probability, 15, 426-432.

Shaked, M. and Shanthikumar, J.G. (2007), Stochastic Orders, Springer, New York.

Zhang, Y., Cai, X., Zhao, P. and Wang, H. (2019), Stochastic Comparisons of Parallel and Series Systems with Heterogeneous Resilience-Scaled Components, Statistics, 53, 126-147.

Zhao, P. and Balakrishnan, N. (2012), Stochastic Comparisons of Largest Order Statistics from Multiple-Outlier Exponential Models, Probability in the Engineering and Informational Sciences, 26, 159-182.

Zhao, P. and Balakrishnan, N. (2015), Comparisons of Largest Order Statistics from Multiple-Outlier Gamma Models, Methodology and Computing in Applied Probability, 17, 617-645. 
Journal of Statistical Sciences, Autumn and Winter, 2021

Vol. 15, No. 2, pp 341-362

DOI: $10.29252 /$ jss.15.2.341

\title{
Stochastic Comparisons of Parallel and Series Systems Com- prising Multiple-Outlier Scale Components
}

\author{
Amini-Seresht, E. ${ }^{1}$ and Barmalzan, G. ${ }^{2}$ \\ ${ }^{1}$ Department of Statistics, Bu-Ali Sina University, Hamedan, Iran. \\ ${ }^{2}$ Department of Statistics, University of Zabol, Zabol, Iran.
}

\begin{abstract}
This paper discusses stochastic comparisons of the parallel and series systems comprising multiple-outlier scale components. Under uncertain conditions on the baseline reversed hazard rate, hazard rate functions and scale parameters, the likelihood ratio, dispersive and mean residual life orders between parallel and series systems are established. We then apply the results for two exceptional cases of the multiple-outlier scale model: gamma and Pareto multiple-outlier components to illustrate the found results.
\end{abstract}

Keywords: Dispersive Order; Likelihood Ratio Order; Series System; Parallel System; Multiple-Outlier Scale Model.

Mathematics Subject Classification (2010): 60E15, 90B25. 